\title{
What MPEG-4 means to me
}

\author{
Murat Kunt
}

The need for a standard in any communication is so obvious that it is not necessary to emphasize it any further. The main problem is to find out what kind of standard we need and how long it should last. Over the past three decades many things went digital: signals, data, telephone and audio to name the major ones. This was a technologydriven evolution, offering many advantages. Short- and long-range robustness, easily controllable precision, repeatability, fidelity, ease of production are so well entrenched that we now take them for granted.

Digital technology also brought the new concept of open system, which is not well accepted and used widely outside research laboratories. We should view an "open system" in its most general sense, as a system that does more than enable a given machine to be connected to others. A truly open system can evolve without questioning the existing structure. At the product level an open system became a must. Customers do not follow any more the fashion of buying a new car radio or a CD reader every month because new models have a few more buttons or functions (read "gadgets") that the previous ones did not have. The pace of progress is much higher than the acceptance of the users. Open system is the obvious solution with which all the new functions can easily be integrated to the existing system. Attempts to make a frozen (closed) system more flexible is called patchwork engineering.
We are all familiar with the well known equation:

$$
\text { MPEG-2 = MPEG-1 + epsilon . }
$$

MPEG-4 is the first standard, leaving aside patchwork and focusing on an open system, the necessary but not sufficient condition for its mid-range survival. The sufficient condition is related to how the openness is managed. If there will be another MPEG- $x$ with $x>4$, this will clearly mean that the sufficient condition has not been satisfied.

\section{Professor Murat Kunt}

Signal Processing Laboratory

Department of Electrical Engineering

Swiss Federal Institute of Technology

$\mathrm{CH}-1015$ Lausanne

Switzerland

Tel.: +41216932626

Fax: +41216932603

e-mail:kunt@epfl.ch or m.kunt@ieee.org 